\title{
Demodulation of intensity and shot noise in the optical heterodyne detection of laser interferometers for gravitational waves
}

\author{
Malik Rakhmanov
}

\begin{abstract}
Demodulation of intensity noise in the optical heterodyne detector is analyzed for application in interferometric gravitational-wave detectors. The correlation function and the power spectral density of the demodulated intensity noise are derived, taking into account the effect of bandpass filtering at the photodiode and an arbitrary demodulation waveform. The analysis includes demodulation of the rf-modulated shot noise as a special case of the intensity noise. For shot-noise-limited detection, the signal-to-noise ratio is found as a function of the modulation parameters, and the optimization of the signal-to-noise ratio with respect to the demodulation phase is described. (C) 2001 Optical Society of America
\end{abstract}

OCIS codes: $\quad 040.2840,030.5260,060.5060,040.5160$.

\section{Introduction}

The heterodyne technique is often used for highsensitivity measurements of an optical phase. The technique is based on coherent detection of the rfmodulated intensity that results from the superposition of laser fields with different frequencies. The $\mathrm{rf}$ modulation shifts the frequency of the measured intensity into the band where the photodetection is quiet and, at best, is limited by shot noise. The result of the photodetection, the rf-modulated photocurrent, is then rectified by a mixer to obtain the low-frequency signal.

Special attention to the shot noise in the optical heterodyne detection has been given in the design of laser interferometers for gravitational-wave detection. The sensitivity of these interferometers in the gravitational-wave detection bandwidth is expected to be limited by shot noise only. Current signal extraction schemes utilized in these interferometers require

When this research was performed, the author was with the Laser Interferometer Gravitational Wave Observatory (LIGO) Project, California Institute of Technology, 1200 East California Boulevard, Pasadena, California 91125. He is now with the Department of Physics, University of Florida, P.O. Box 118440, Gainesville, Florida 32611 and can be reached at malik@ phys.ufl.edu.

Received 15 August 2000; revised manuscript received $27 \mathrm{Au}-$ gust 2001.

0003-6935/01/366596-10\$15.00/0

(C) 2001 Optical Society of America rf modulation, which inevitably makes the shot noise nonstationary and therefore difficult to analyze.

One of the earliest studies of shot noise in prototype gravitational-wave detectors was done by Niebauer et al. ${ }^{1}$ who found that nonstationary shot noise has frequency correlations that can increase or decrease the power spectral density of the demodulated noise. At about the same time, Meers and Strain ${ }^{2}$ analyzed the generation of shot noise in various modulation schemes. In the experiments performed by Mio and Tsubono ${ }^{3}$ and also by Gray et al. ${ }^{4}$ the frequency correlations of the nonstationary shot noise were studied indirectly, through the phase dependence of the shot-noise power spectral density. Arbitrary filtering of the nonstationary shot noise was discussed by Winzer, ${ }^{5}$ who showed that noise variance depends on the transfer function of the filter. Recently Lyons et al. ${ }^{6}$ calculated the shot noise in interferometric gravitational-wave detectors taking into account the effect of Fabry-Perot cavities in the interferometer arms. In all these studies, the main quantity of interest is the power spectral density of shot noise after demodulation.

So far, calculations of the power spectra of shot noise in the prototype interferometers did not take into account the effects of bandpass filtering of the $\mathrm{rf}$ photocurrent. Usually, a sine-wave demodulation is assumed instead of the more realistic square-wave demodulation. Moreover, high-order harmonics of the rf-modulated photocurrent were often neglected. This simplified approach can be sufficient for approximate calculations of the shot-noise power spectral 
density. However, for accurate predictions and computer modeling of the shot noise, a rigorous analysis is needed. Such a rigorous analysis is presented in this paper, which describes demodulation of the rfmodulated intensity and shot noise in the heterodyne detection schemes of interferometric gravitationalwave detectors.

In this paper the effects of bandpass filtering of the rf photocurrent are studied, and demodulation with an arbitrary waveform is described. The mathematical formalism allows us to calculate the correlation function and the power spectral density of the intensity and shot noise after demodulation. Although these calculations are developed for applications to interferometric gravitational-wave detectors, the approach is general and can be used to assess noise performance of other measurement devices that utilize optical heterodyne mixing.

\section{Signal Extraction}

\section{A. Photodetection of Modulated Intensity}

The optical heterodyne detection scheme, which is currently used in interferometric gravitationalwave detectors, ${ }^{7-9}$ is based on the Pound-Drever signal extraction method. ${ }^{10}$ Such heterodyne detection is schematically shown in Fig. 1 and can be briefly described as follows. The rf oscillator serves as a reference source for both modulation of the laser field and demodulation of the rf photocurrent. (The modulation can be done, for example, by an electro-optic modulator or an acousto-optic deflector.) The laser field propagates in the interferometer and is detected at the photodiode. The output of the photodiode is then rectified by the mixer. Important elements of this signal extraction scheme are the bandpass filter and the phase shifter. Their effect on the power spectral density of noise is described in this paper.

The frequency of the rf oscillator defines the modulation frequency $\Omega$ and the frequencies of the modulation harmonics:

$$
\Omega_{n}=n \Omega,
$$

where $n$ is an integer. The modulated intensity can be described by a Fourier series:

$$
P(t)=\sum_{n=-\infty}^{\infty} P_{n} \exp \left(i \Omega_{n} t\right),
$$

where $P_{n}$ is the complex amplitude of the $n$th harmonic. For intensity to be a real quantity, the coefficients $P_{n}$ must satisfy the condition

$$
P_{-n}=P_{n}^{*}
$$

where the asterisk denotes complex conjugation. The photodiode generates a photocurrent $I(t)$ that is proportional to the intensity:

$$
I(t)=\chi P(t),
$$

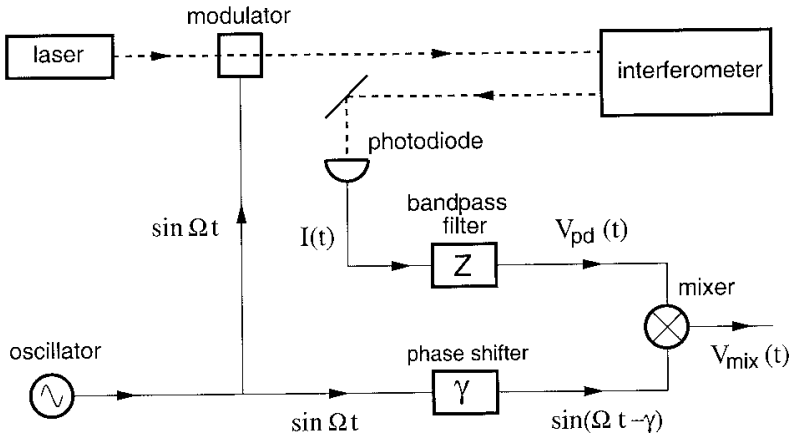

Fig. 1. Example of the optical heterodyne detection scheme.

where $\chi$ is the photodiode responsivity (typically 0.2 $0.7 \mathrm{~A} / \mathrm{W}$ for a wavelength of $1 \mu \mathrm{m})$. The photocurrent that corresponds to modulated intensity also becomes modulated:

$$
I(t)=\sum_{n=-\infty}^{\infty} I_{n} \exp \left(i \Omega_{n} t\right),
$$

where $I_{n}=\chi P_{n}$. At this point, the intensity and the photocurrent are assumed to be deterministic quantities; the associated random processes are introduced below as noise.

Assume that the photodetection and subsequent demodulation take place within a finite time $T$ (observation time), which is much greater than the modulation period. Finite duration signals can be analyzed using the finite-time Fourier transformation. (In this paper, all Fourier transforms are defined as finite-time Fourier transforms.) For example, the Fourier transform of the modulated photocurrent in Eq. (5) is

$$
\tilde{I}(\omega)=2 \pi \sum_{n=-\infty}^{\infty} I_{n} \delta_{T}\left(\omega-\Omega_{n}\right),
$$

where $\delta_{T}(\omega)$ represents the finite-time $\delta$ function. The definitions of the finite-time Fourier transformation, its inverse, and the $\delta$ function are given in Appendix A.

Thus far we assume that the coefficients $P_{n}$ and $I_{n}$ are constant. In general, these coefficients can vary with time, deviating slightly from their average (constant) values. In this case, Fourier transform of the photocurrent becomes

$$
\tilde{I}(\omega)=\sum_{n=-\infty}^{\infty} \tilde{I}_{n}\left(\omega-\Omega_{n}\right),
$$

where $\tilde{I}_{n}(\omega)$ are the finite-time Fourier transforms of the coefficients $I_{n}(t)$.

If the rate of the variations is much less than the modulation frequency, the coefficients $I_{n}(t)$ change little during one modulation period. Then the Fourier transform of the photocurrent consists of a series of narrow peaks at frequencies of the modulation harmonics, as shown in Fig. 2. As a result, the amplitude of the Fourier transform of the photocurrent in the vicinity of the $n$th peak is largely defined by the 


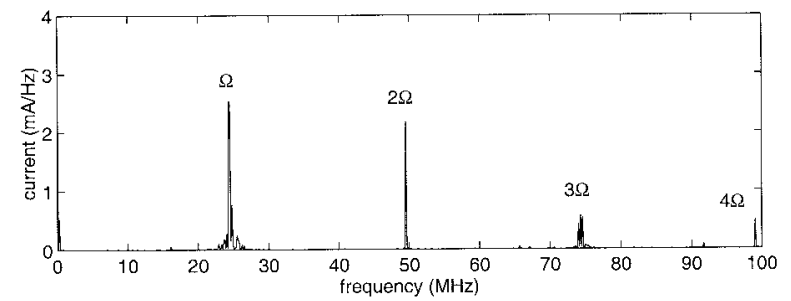

Fig. 2. Typical spectrum of the rf-modulated photocurrent at the output of the LIGO rf photodetector. The modulation frequency $\Omega$ is approximately $24.7 \mathrm{MHz}$.

$n$th harmonic. Then the following approximation holds:

$$
\tilde{I}\left(\Omega_{n}+\omega\right) \approx \tilde{I}_{n}(\omega),
$$

provided that the frequency offset $\omega$ is small $(|\omega| \ll$ $\Omega$ ).

\section{B. Bandpass Filtering of the Radio Frequency Photocurrent}

In the heterodyne detection based on the PoundDrever method, the signal is obtained from the first harmonic, $I_{1}$, of the modulated photocurrent. The higher-order harmonics, $I_{2}, I_{3} \ldots$, as well as the dc current, $I_{0}$, mostly contribute to noise. These harmonics can be suppressed by a bandpass filter that is placed immediately behind the photodiode or is integrated into the photodiode's circuit. We denote the impedance of the photodiode circuit with the bandpass filter by $\tilde{Z}(\omega)$. Then the voltage output of the photodiode is

$$
\tilde{V}_{\mathrm{pd}}(\omega)=\tilde{Z}(\omega) \tilde{I}(\omega) .
$$

Note that the impedance satisfies the condition

$$
\tilde{Z}(-\omega)=\tilde{Z}(\omega)^{*},
$$

which guarantees that $V_{\mathrm{pd}}(t)$ is a real quantity. An example of the bandpass filter is shown in Fig. 3. The peak of the transfer function is matched to the modulation frequency $\Omega$, and the width of the peak $\Delta \Omega$ defines the bandwidth of the filter.

The magnitude of the signal is mostly defined by the value of the impedance at the peak:

$$
\tilde{Z}(\Omega)=R \exp (-i \beta),
$$

where $R$ is the magnitude and $\beta$ is the phase lag. (The impedance shown in Fig. 3 has a phase lag of approximately 130 deg.) To avoid distortion of the signal, the impedance must be sufficiently flat near the peak, and the width of the peak must be greater than the bandwidth of the signal. Then the impedance would be approximately constant:

$$
\tilde{Z}(\Omega \pm \omega) \approx \tilde{Z}(\Omega)
$$

for typical frequencies of the signal $\omega$.
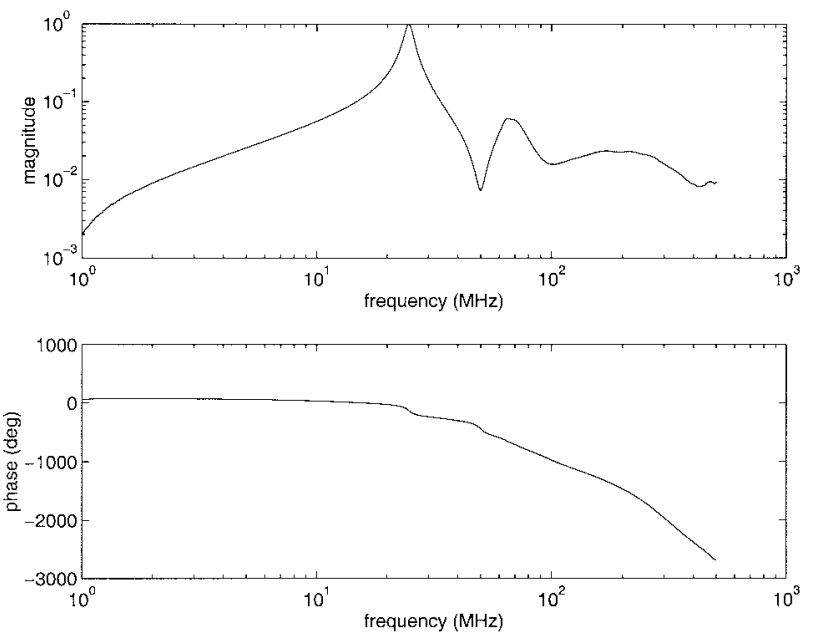

Fig. 3. Transfer function of a typical LIGO $\mathrm{rf}$ photodiode. The upper plot shows the magnitude of the transfer function; the lower plot shows its phase. The peak of the transfer function is 24.7 $\mathrm{MHz}$, and the width of the peak is $3.8 \mathrm{MHz}$. The notch at 49.4 $\mathrm{MHz}$ was introduced to suppress the second harmonic of the rf photocurrent.

\section{Demodulation of the Signal}

The filtered rf signal is rectified by the mixer (Fig. 1) to obtain a low-frequency signal. The output of the mixer can be described by the product

$$
V_{\text {mix }}(t)=D(t) V_{\mathrm{pd}}(t),
$$

where $D(t)$ is the demodulation wave. The corresponding quantity in frequency domain is given by the convolution

$$
\tilde{V}_{\text {mix }}(\omega)=\int_{-\infty}^{\infty} \tilde{D}\left(\omega-\omega^{\prime}\right) \tilde{V}_{\mathrm{pd}}\left(\omega^{\prime}\right) \frac{\mathrm{d} \omega^{\prime}}{2 \pi} .
$$

The low-frequency component $\tilde{V}(\omega)$ of the mixer output constitutes the signal in the optical heterodyne detection:

$$
\tilde{V}(\omega)=\left.\tilde{V}_{\text {mix }}(\omega)\right|_{|\omega| \ll \Delta \Omega} .
$$

In practice, separation of the low-frequency component from the mixer output is done by a low-pass filter with the cutoff frequency being less than the modulation frequency. Being a part of the postprocessing electronics such a filter affects both the signal and the noise equally and is therefore excluded from the present analysis.

Often the demodulation waveform is assumed to be sinusoidal:

$$
D(t)=\sin (\Omega t-\gamma),
$$

where $\gamma$ is an adjustable phase set by the phase shifter, shown in Fig. 1. Although this choice of the demodulation waveform greatly simplifies calculations, it is not usually realized in experiments. The demodulation waveform of a typical rf mixer (fullwave diode bridge) is very close to the square wave 
with an amplitude of 1 . Such a waveform can be described by the Fourier series

$$
D(t)=\frac{4}{\pi} \sum_{n=1,3,5, \ldots} \frac{1}{n} \sin n(\Omega t-\gamma) .
$$

In general, the demodulation waveform can be arbitrary:

$$
D(t)=-i \sum_{n=-\infty}^{\infty} d_{n} \exp [i n(\Omega t-\gamma)],
$$

where the coefficients $d_{n}$ satisfy the condition $d_{-n}=$ $-d_{n}{ }^{*}$. One can obtain the sinusoidal demodulation from the general form in Eq. (18) by setting all the coefficients to zero except for

$$
d_{1}=-d_{-1}=1 / 2 .
$$

The square-wave demodulation can be obtained from the general form by setting

$$
d_{n}=\left\{\begin{array}{ll}
0, & n=\text { even } \\
2 /(\pi n), & n=\text { odd }
\end{array} .\right.
$$

Fourier transform of the demodulation waveform is a sum of $\delta$ functions at frequencies of the modulation harmonics:

$$
\tilde{D}(\omega)=-2 \pi i \sum_{n=-\infty}^{\infty} d_{n} \exp (-i n \gamma) \delta_{T}\left(\omega-\Omega_{n}\right) .
$$

Correspondingly, the mixer output in Eq. (14) also becomes the sum:

$$
\tilde{V}_{\text {mix }}(\omega)=-i \sum_{n=-\infty}^{\infty} d_{n} \exp (-i n \gamma) \tilde{Z}\left(\omega-\Omega_{n}\right) \tilde{I}\left(\omega-\Omega_{n}\right) \text {. }
$$

To evaluate the integral in Eq. (14), we treated the finite-time $\delta$ function as the usual Dirac $\delta$ function. This is a valid approximation as long as the observation time is much greater than the modulation period. ${ }^{11}$

An explicit formula for the signal can be obtained from Eq. (22) by use of the low-frequency approximation (8):

$$
\tilde{V}(\omega) \approx-i \sum_{n=-\infty}^{\infty} d_{n} \exp (-i n \gamma) \tilde{Z}\left(\omega-\Omega_{n}\right) \tilde{I}_{-n}(\omega) .
$$

This formula shows that the $n$th harmonic of the $\mathrm{rf}$ photocurrent $\left(\tilde{I}_{-n}\right)$ beats against the $n$th harmonic of the demodulation waveform $\left(d_{n}\right)$ to produce the lowfrequency signal. Usually the first $(n= \pm 1)$ harmonic carries information about the optical phase. Other harmonics can introduce errors in the signal extraction and therefore must be suppressed. The suppression must precede the mixing and is the main function of the bandpass filter.

The suppression of high-order harmonics of the rf photocurrent requires that

$$
\left|\tilde{Z}\left(\omega \pm \Omega_{n}\right)\right| \ll R,
$$

for $n=2,3, \ldots$ In particular, a notch filter at frequency $2 \Omega$ can be incorporated into the bandpass filter (Fig. 3) to obtain a strong suppression of the second harmonic of the modulated photocurrent. As a result of this narrow-band filtering, the signal becomes proportional to the first harmonic of the photocurrent

$$
\begin{aligned}
\tilde{V}(\omega) \approx & -i d_{-1} \exp (i \gamma) \tilde{Z}(\omega+\Omega) \tilde{I}_{1}(\omega) \\
& -i d_{1} \exp (-i \gamma) \tilde{Z}(\omega-\Omega) \tilde{I}_{-1}(\omega),
\end{aligned}
$$

and is therefore largely independent of the demodulation waveform.

For typical frequencies of the signal, the impedance can be approximated as a constant [approximation (12)], and the signal becomes

$$
\begin{aligned}
\tilde{V}(\omega)= & -i d_{-1} \exp [i(\gamma-\beta)] R \tilde{I}_{1}(\omega) \\
& -i d_{1} \exp [-i(\gamma-\beta)] R \tilde{I}_{-1}(\omega) .
\end{aligned}
$$

The corresponding equation in the time domain is

$$
V(t)=-2 d_{1} R \operatorname{Im}\left\{\exp [i(\gamma-\beta)] I_{1}(t)\right\},
$$

where we assume that $d_{1}$ is real and therefore $d_{-1}=$ $-d_{1}$, as is the case for sinusoidal or square-wave demodulation.

The initial phase of the modulation can be chosen so that $I_{1}(t)$ is a real function. ${ }^{12}$ In this case

$$
\begin{gathered}
V(t)=-2 d_{1} R I_{1}(t) \sin (\gamma-\beta), \\
\tilde{V}(\omega)=-2 d_{1} R \tilde{I}_{1}(\omega) \sin (\gamma-\beta) .
\end{gathered}
$$

The optimal demodulation corresponds to the maximum of the absolute value of the signal. It can be achieved by matching the phase of the demodulation waveform $D(t)$ to the phase of the photodiode's output $V_{\text {pd }}(t)$ according to

$$
\gamma=\beta+\pi / 2+\pi N,
$$

where $N$ is an integer. This condition can be met by adjustment of the demodulation phase $\gamma$ at the phase shifter (Fig. 1). Such an optimization is applicable if the noise does not depend on the demodulation phase or if the signal is much greater than the noise. Otherwise, one can optimize the demodulation phase by maximizing the signal-to-noise ratio as described below.

\section{Intensity and Shot Noise}

\section{A. Demodulation of Intensity Noise}

The common noise source that affects the measurements based on photodetection of the laser fields is the intensity noise. An arbitrary intensity noise is caused by random fluctuations of the laser power $\delta P(t)$ at the photodiode. The intensity noise gives rise to the noise in the photocurrent:

$$
\delta I(t)=\chi \delta P(t) .
$$


Therefore the noisy photocurrent can be represented as the sum:

$$
J(t)=I(t)+\delta I(t),
$$

where $I(t)$ is the average photocurrent,

$$
I(t)=\mathbf{E}[J(t)],
$$

and $\delta I(t)$ is the noise with zero mean. In this representation, $\delta I(t)$ corresponds to the raw (unfiltered) intensity noise. The expectation value $\mathbf{E}[J(t)]$ implies an ensemble average of the photocurrent. The ensemble average can be implemented as an average over a set of traces of the photocurrent recorded with a storage oscilloscope. It can also be implemented as the time average according to the ergodic theorem. ${ }^{11}$ The time interval for such an average must be as large as possible but much less than the period of the rf modulation.

The noise in the photocurrent is characterized by its correlation function:

$$
C_{I}\left(t, t^{\prime}\right) \equiv \mathbf{E}\left[\delta I(t) \delta I\left(t^{\prime}\right)\right],
$$

which is defined mostly by the laser and the electrooptic devices along the beam propagation. The correlation function in the frequency domain can be defined as

$$
\tilde{C}_{I}\left(\omega, \omega^{\prime}\right)=\mathbf{E}\left[\delta \tilde{I}(\omega) \delta \tilde{I}\left(\omega^{\prime}\right)^{*}\right],
$$

which is equivalent to the finite-time Fourier transformation

$$
\begin{aligned}
\tilde{C}_{I}\left(\omega, \omega^{\prime}\right)= & \int_{-T / 2}^{T / 2} \int_{-T / 2}^{T / 2} \exp \left[-i\left(\omega t-\omega^{\prime} t^{\prime}\right)\right] \\
& \times C_{I}\left(t, t^{\prime}\right) \mathrm{d} t \mathrm{~d} t^{\prime} .
\end{aligned}
$$

Demodulation of the intensity noise can be described similarly to the demodulation of the signal. The noise passes through the bandpass filter and is then rectified by the mixer. The noise at the output of the mixer is

$$
\delta \tilde{V}(\omega)=-i \sum_{n=-\infty}^{\infty} d_{n} \exp (-i n \gamma) \tilde{Z}\left(\omega-\Omega_{n}\right) \delta \tilde{I}\left(\omega-\Omega_{n}\right) .
$$

Thus the correlation function of the demodulated noise can be defined as

$$
\tilde{C}_{V}\left(\omega, \omega^{\prime}\right)=\mathbf{E}\left[\delta \tilde{V}(\omega) \delta \tilde{V}\left(\omega^{\prime}\right)^{*}\right] .
$$

Combining Eqs. (37) and (38), we obtain a formula for the correlation function of the intensity noise at the mixer output:

$$
\begin{aligned}
\tilde{C}_{V}\left(\omega, \omega^{\prime}\right)= & \sum_{m=-\infty}^{\infty} \sum_{n=-\infty}^{\infty} d_{m} d_{n}{ }^{*} \exp [-i(m-n) \gamma] \\
& \times \tilde{Z}\left(\omega-\Omega_{m}\right) \tilde{Z}\left(\omega^{\prime}-\Omega_{n}\right)^{*} \\
& \times \tilde{C}_{I}\left(\omega-\Omega_{m}, \omega^{\prime}-\Omega_{n}\right)
\end{aligned}
$$

This correlation function depends on the bandpass filter and the demodulation waveform. The power spectral density of the demodulated noise can be derived from this correlation function according to the definition

$$
S_{V}(\omega)=\lim _{T \rightarrow \infty}\left[\frac{\tilde{C}_{V}(\omega, \omega)}{T}\right],
$$

which applies to both stationary and nonstationary random processes. ${ }^{13}$

In the presence of narrow-band filtering [inequality (24)], the intensity noise outside the passband of the filter is greatly suppressed. In this case, the formulas for the demodulated noise [Eq. (37)] and its correlation function [Eq. (39)] can be simplified. Specifically the demodulated noise is defined mostly by the first harmonic of the photocurrent:

$$
\begin{aligned}
\delta \tilde{V}(\omega)= & -i d_{-1} \exp (i \gamma) \tilde{Z}(\omega+\Omega) \delta \tilde{I}(\omega+\Omega) \\
& -i d_{1} \exp (-i \gamma) \tilde{Z}(\omega-\Omega) \delta \tilde{I}(\omega-\Omega) .
\end{aligned}
$$

As a result, all the modulation harmonics, except for the first one $(m, n= \pm 1)$, can be omitted from the sum in Eq. (39). The same result can be obtained if impedance $\tilde{Z}(\omega)$ is broadband but the demodulation wave is sinusoidal. Therefore, broadband photodetection with sine-wave demodulation is equivalent to narrow-band photodetection with square-wave demodulation. ${ }^{14}$ Moreover, the presence of the narrow-band filter makes the demodulation essentially independent of waveform $D(t)$. In this case, the demodulation depends only on the coefficient $d_{1}$. For example, the square-wave demodulation of amplitude 1 is equivalent to sine-wave demodulation of amplitude $4 / \pi$.

\section{B. Stationary Intensity Noise}

Consider a situation when the intensity noise is stationary and therefore its correlation function has the form

$$
C_{I}\left(t, t^{\prime}\right)=C_{I}\left(t-t^{\prime}\right) .
$$

Fourier transformation of this correlation function is diagonal:

$$
\tilde{C}_{I}\left(\omega, \omega^{\prime}\right)=2 \pi S_{I}(\omega) \delta_{T}\left(\omega-\omega^{\prime}\right),
$$

where $S_{I}(\omega)$ is the Fourier transform of $C_{I}(t)$. According to the Wiener-Khinchin theorem, $S_{I}(\omega)$ is the power spectral density of the intensity noise. The theorem does not apply to nonstationary noise, in which case a more general definition [Eq. (40)] can be used to calculate the power spectral density.

The correlation function of the noise at the output of the mixer can be found from Eqs. (39) and (43). The demodulated noise is also stationary:

$$
\tilde{C}_{V}\left(\omega, \omega^{\prime}\right)=2 \pi S_{V}(\omega) \delta_{T}\left(\omega-\omega^{\prime}\right)
$$


and its power spectral density $S_{V}(\omega)$ is given by

$$
S_{V}(\omega)=\sum_{n=-\infty}^{\infty}\left|d_{n}\right|^{2}\left|\tilde{Z}\left(\omega-\Omega_{n}\right)\right|^{2} S_{I}\left(\omega-\Omega_{n}\right) .
$$

Thus we see that the power of the demodulated intensity noise combines the power of the filtered intensity noise from all the modulation harmonics.

An ideal narrow-band filter would greatly suppress the noise from all harmonics except the first one. Then all the high-order terms in the sum [Eq. (45)] can be neglected and the power spectral density becomes

$$
\begin{aligned}
S_{V}(\omega)= & \left|d_{1}\right|^{2}|\tilde{Z}(\omega-\Omega)|^{2} S_{I}(\omega-\Omega) \\
& +\left|d_{1}\right|^{2}|\tilde{Z}(\omega+\Omega)|^{2} S_{I}(\omega+\Omega) .
\end{aligned}
$$

For low frequencies $(\omega \ll \Delta \Omega)$, the power spectral density becomes constant:

$$
S_{V}(\omega) \approx 2\left|d_{1}\right|^{2} R^{2} S_{I}(\Omega) .
$$

This relation shows that the noise at the output of the mixer is due mostly to the intensity noise at the modulation frequency. Usually, the power of the raw intensity noise diminishes as the frequency of the noise increases,

$$
S_{I}(\Omega) \ll S_{I}(0) .
$$

Thus, in an ideal situation, the intensity noise becomes greatly reduced by the heterodyne detection.

In practice, the bandpass filter transmits some of the noise at frequencies of the modulation harmonics. This can lead to a substantial difference between the ideal power spectral density [Eq. (46)] and the morerealistic power spectral density given by Eq. (45). To avoid the accumulation of noise from high-order harmonics, these harmonics must be suppressed by the bandpass filter.

\section{Nonstationary Intensity Noise}

Consider now a situation in which the intensity noise is nonstationary. For simplicity, we assume that strong narrow-band filtering takes place in the photodiode circuit. In this case, the correlation function of the demodulated noise is given by Eq. (39) with $m$, $n= \pm 1$, and the power spectral density is given by

$$
\begin{aligned}
S_{V}(\omega)= & \left|d_{1}\right|^{2}|\tilde{Z}(\omega-\Omega)|^{2} S_{I}(\omega-\Omega) \\
& +\left|d_{1}\right|^{2}|\tilde{Z}(\omega+\Omega)|^{2} S_{I}(\omega+\Omega) \\
& -2\left|d_{1}\right|^{2} \operatorname{Re}\{\exp (2 i \gamma) \\
& \left.\times \tilde{Z}(\omega-\Omega) * \tilde{Z}(\omega+\Omega) Q_{I}(\omega)\right\} .
\end{aligned}
$$

Here we introduced a new quantity,

$$
Q_{I}(\omega)=\lim _{T \rightarrow \infty}\left[\frac{\tilde{C}_{I}(\omega+\Omega, \omega-\Omega)}{T}\right],
$$

which is related to the nonstationary property of the noise. $\left(Q_{I}=0\right.$ if the noise is stationary. $)$

The first two terms on the right-hand side of Eq. (49) are necessarily positive and represent the power of the uncorrelated noise. The last term represents the power of noise from its frequency correlations. This additional contribution can be both positive and negative and can therefore increase or decrease the total power of the demodulated noise.

In the low-frequency approximation [Eq. (12)] the demodulated noise becomes

$$
\begin{aligned}
S_{V}(\omega)= & 2\left|d_{1}\right|^{2} R^{2} S_{I}(\Omega) \\
& -2\left|d_{1}\right|^{2} R^{2} \operatorname{Re}\left\{\exp [2 i(\beta-\gamma)] Q_{I}(0)\right\} .
\end{aligned}
$$

Therefore, accurate estimations of the power spectral density of the nonstationary intensity noise at the output of the mixer require measurements of both quantities: $S_{I}(\Omega)$ and $Q_{I}(0)$.

\section{Nonstationary Shot Noise}

The heterodyne technique provides strong suppression of laser intensity noise if a sufficiently high modulation frequency is chosen. At this frequency, the photodetection becomes quiet and ideally is limited by shot noise only.

The shot noise is generated during photodetection and is largely defined by the average photocurrent $I(t)$. In the optical heterodyne detection, the average photocurrent varies with time causing the shot noise to be inherently nonstationary. The nonstationary shot noise is characterized by its correlation function

$$
\tilde{C}_{I}\left(\omega, \omega^{\prime}\right)=q \tilde{I}\left(\omega-\omega^{\prime}\right),
$$

where $q$ is the electron charge and $\tilde{I}(\omega)$ is the Fourier transform of the average photocurrent. (Derivation of this formula is given in Appendix B.)

In the following analysis, we neglect the time dependence of the amplitudes of the modulated photocurrent and assume that all the $I_{n}$ are constant. Then the correlation function of the nonstationary shot noise is given by

$$
\tilde{C}_{I}\left(\omega, \omega^{\prime}\right)=2 \pi q \sum_{n=-\infty}^{\infty} I_{n} \delta_{T}\left(\omega-\omega^{\prime}-\Omega_{n}\right)
$$

Substituting this equation into the general formula [Eq. (39)], we find that the correlation function of the demodulated noise is diagonal:

$$
\tilde{C}_{V}\left(\omega, \omega^{\prime}\right)=2 \pi S_{V}(\omega) \delta_{T}\left(\omega-\omega^{\prime}\right),
$$

which means that the demodulated shot noise is stationary and that its power spectral density is given by

$$
\begin{aligned}
S_{V}(\omega)= & \sum_{m=-\infty}^{\infty} \sum_{n=-\infty}^{\infty} d_{m} d_{n}{ }^{*} \exp [-i(m-n) \gamma] \\
& \times q I_{n-m} \tilde{Z}\left(\omega-\Omega_{m}\right) \tilde{Z}\left(\omega-\Omega_{n}\right)^{*} .
\end{aligned}
$$

This formula describes accumulation of the shotnoise power from all the harmonics of the modulated photocurrent. (Derivation of this formula is given in Appendix A.) 
In the presence of a strong narrow-band filter, the power spectral density becomes

$$
\begin{aligned}
S_{V}(\omega)= & q d_{1}^{2} I_{0}\left[|\tilde{Z}(\omega-\Omega)|^{2}+|\tilde{Z}(\omega+\Omega)|^{2}\right] \\
& -2 q d_{1}{ }^{2} \operatorname{Re}\left\{I_{2} \tilde{Z}(\omega-\Omega) * \tilde{Z}(\omega+\Omega) \exp (2 i \gamma)\right\} .
\end{aligned}
$$

Such a power spectral density has a characteristic shape of the bandpass filter. This observation can be used to distinguish the shot noise at the output of the mixer from other sources of noise that could be added by the postprocessing electronics.

The power spectral density [Eq. (56)] can also be derived from the general formula for the intensity noise [Eq. (49)] by noting that

$$
\begin{aligned}
& S_{I}(\omega)=q I_{0}, \\
& Q_{I}(\omega)=q I_{2} .
\end{aligned}
$$

Equation (57) is the well-known Schottky formula. Equation (58) shows that the noise energy that is due to the frequency correlations is proportional to the amplitude of the second harmonic of the photocurrent.

In the low-frequency approximation [Eq. (12)], the power spectral density becomes

$$
S_{V}(\omega) \approx 2 q d_{1}^{2} R^{2}\left(I_{0}-\operatorname{Re}\left\{I_{2} \exp [2 i(\gamma-\beta)]\right\}\right) .
$$

The two parts of this power spectral density (proportional to $I_{0}$ and $I_{2}$ ) correspond to the stationary and the nonstationary components of the shot noise. Note that coefficient $I_{0}$ is real but coefficient $I_{2}$, in general, is complex.

The formulas for the power spectral density of the demodulated shot noise, Eq. (56) and approximation (59), reveal an interesting difference between the deterministic photocurrent and the random noise. The notch filter, which suppresses the $I_{2}$ component of the average photocurrent, has no effect on the $I_{2}$ component of the shot noise. This peculiar fact can be explained by the observation that the $I_{2}$ component of the demodulated shot noise does not originate from the $2 \Omega$ component of the modulated photocurrent; it originates from the correlations between the noise components at all frequencies separated by $2 \Omega$.

The largest amplitude of the second harmonic of the photocurrent corresponds to the special case

$$
I_{2}=I_{0} / 2,
$$

which appears during maximum (100\%) modulation of the photocurrent:

$$
I(t)=I_{0}(1+\cos 2 \Omega t) .
$$

The power spectral density of the demodulated shot noise for such a photocurrent is given by

$$
S_{V}(\omega) \approx 2 q d_{1}^{2} R^{2} I_{0}\left[1-\frac{1}{2} \cos 2(\gamma-\beta)\right] .
$$

A similar expression for the power spectral density was found by Niebauer et al. ${ }^{1}$ According to approximation (62), the total power of the demodulated shot noise can be increased or decreased by $50 \%$ by changing in the demodulation phase. This observation is valid only for the ideal (maximum) modulation of the photocurrent [Eq. (61)]. It also assumes that narrow-band filtering takes place at the photodiode. If these conditions are not satisfied, a more general formula [Eq. (55)] can be used to calculate the power spectral density of the demodulated shot noise.

\section{E. Optimal Demodulation Phase}

When the detection is limited by the intensity or shot noise, both the signal and the noise at the output of the mixer depend on the demodulation phase. This phase dependence strongly affects the signal-to-noise ratio (SNR). Therefore, an optimization with respect to the demodulation phase is needed.

If signal $V(t)$ is a long-lasting periodic function of time, the SNR is defined as

$$
(\mathrm{SNR})^{2}=\frac{\overline{|V(t)|^{2}}}{S_{V}\left(\omega_{g}\right) \Delta \omega},
$$

where $\omega_{g}$ is the frequency of the signal and $\Delta \omega=$ $2 \pi / T$ is the resolution bandwidth of the measurement. If the signal is of short duration compared to the observation time (burst), a different definition of the SNR is used. Namely, for bursts with known waveforms, Wiener optimal filtering leads to the following SNR ${ }^{15}$ :

$$
(\mathrm{SNR})^{2}=\int_{-\infty}^{\infty} \frac{|\tilde{V}(\omega)|^{2}}{S_{V}(\omega)} \frac{\mathrm{d} \omega}{2 \pi},
$$

where $\tilde{V}(\omega)$ is the Fourier transform of the signal and $S_{V}(\omega)$ is the power spectral density of the demodulated noise. In either case, periodic signals or bursts, the SNR is proportional to the following function of the demodulation phase:

$$
y=\frac{\sin ^{2}(\gamma-\beta)}{1-\operatorname{Re}\{z \exp [2 i(\gamma-\beta)]\}} .
$$

The complex parameter $z$ is defined as

$$
z=\frac{Q_{I}(0)}{S_{I}(\Omega)}
$$

and is the measure of nonstationarity of the intensity noise. Note that the SNR in Eq. (64) does not depend on the demodulation waveform. This result is a consequence of the narrow-band filtering at the photodiode, which implies that no optimization with respect to the demodulation waveform is necessary.

The optimal demodulation phase corresponds to a maximum $y$ factor as a function of $\gamma$ and can be found from the condition

$$
\partial y / \partial \gamma=0
$$




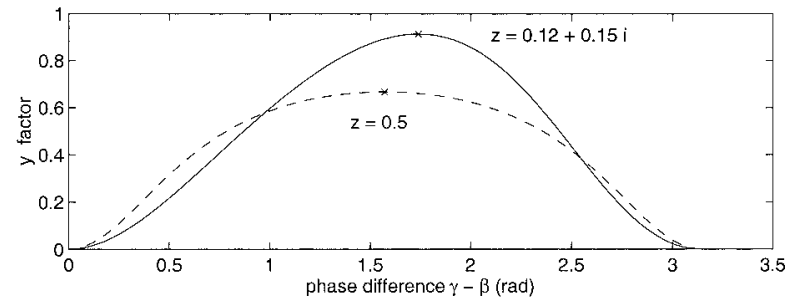

Fig. 4. Function of the demodulation phase represented by the $y$ factor. The dashed curve corresponds to ideal (100\% modulation) detection; the solid curve corresponds to detection with the parameters of the LIGO interferometers. The optimal demodulation points are marked by $\mathrm{x}$.

The solution to Eq. (67) is given by

$$
\gamma=\beta+\arctan \left(\frac{\operatorname{Re}\{z\}-1}{\operatorname{Im}\{z\}}\right)+\pi N,
$$

where $N$ is an integer. Such a demodulation phase differs from the one that is obtained by maximization of the signal alone [Eq. (30)]. The corresponding optimal value of the $y$ factor is given by

$$
y_{\mathrm{opt}}=\frac{1-\operatorname{Re}\{z\}}{1-|z|^{2}} .
$$

For small nonstationary noise, $(|z| \ll 1)$, the optimal value of the $y$ factor becomes independent of the parameters of the heterodyne detection:

$$
y_{\text {opt }} \approx 1 \text {. }
$$

When the detection is limited by shot noise, complex parameter $z$ is defined by the zeroth and second harmonics of the modulated photocurrent:

$$
z=I_{2} / I_{0} \text {. }
$$

Examples of the $y$ factor as a function of the demodulation phase are shown in Fig. 4. The two curves in the figure correspond to the ideal modulation $(z=$ $1 / 2)$ and the more-realistic modulation $(z=0.12 \pm$ $0.15 i$ ), which is calculated with the parameters of the Laser Interferometer Gravitational Wave Observatory (LIGO) 4 -km interferometers. ${ }^{12}$

In general, the value of parameter $z$ is not known beforehand. Therefore it is worthwhile to find the range of possible values of the optimal $y$ factor. Consider the average photocurrent $I(t)$. The condition for unidirectional flow, $I(t)>0$, implies that the magnitude of the second harmonic of the modulated photocurrent must be limited,

$$
\left|I_{2}\right| \leq I_{0} / 2
$$

which is equivalent to

$$
|z| \leq 1 / 2 \text {. }
$$

This condition represents a circle in the complex $z$ plane. Then analytic function $y_{\text {opt }}(z)$ achieves its extremum values on the boundary of the circle, namely, at the points

$$
z= \pm 1 / 2
$$

These boundary points define the range for the optimal $y$ factor:

$$
2 / 3 \leq y_{\text {opt }} \leq 2 \text {. }
$$

The lower limit, $y_{\text {opt }}=2 / 3$, appeared in the calculations by Niebauer et al. ${ }^{1}$ To the best of my knowledge, the upper limit, $y_{\mathrm{opt}}=2$, has not yet been analyzed. In general, the optimal value of the $y$ factor depends on the optical configuration of the heterodyne detector and appears somewhere between $2 / 3$ and 2 .

\section{Conclusion}

An essential part of the heterodyne detector is the bandpass filter that suppresses the higher-order harmonics of the modulated photocurrent. The result of bandpass filtering is that both the signal and the noise depend only on the first harmonics of the demodulation waveform (their magnitude is proportional to $\left.d_{1}\right)$. Moreover, the SNR becomes independent of $d_{1}$, and no optimization with respect to the demodulation waveform is necessary.

The power spectral density of the demodulated intensity noise, $S_{V}(\omega)$, depends on both the power spectral density, $S_{I}(\omega)$, and the correlation function, $Q_{I}(\omega)$, of the raw intensity noise. Thus accurate predictions of the demodulated noise at the output of the mixer require measurements of both the power spectral density and the correlation function of the intensity noise at the photodiode.

High sensitivity measurements of the optical phase based on the heterodyne technique, in principle, are limited by shot noise only. Reaching the shot-noise limit is a target for many such measurements, especially for those that will be performed with the new laser gravitational-wave detectors. To verify that the sensitivity has reached the desired shot-noise limit, one can apply various tests to the power spectral density of the measured noise. In addition to the usual test of linearity with respect to the input laser power, one can measure the frequency dependence of the power spectral density or its dependence on the demodulation phase. These functional dependences have been described in this paper.

If detection is limited by shot noise, the SNR depends on the demodulation phase. Optimization with respect to this phase is necessary to achieve the best sensitivity. For optimal demodulation phase, the values of the $y$ factor are limited to the interval $[2 / 3,2]$. Further improvement of the SNR can be done if one increases the value of the $y$ factor and ultimately reaches the upper limit: $y_{\text {opt }}=2$.

\section{Appendix A. Finite-Time Fourier Transform}

The finite-time Fourier transformation of a timedomain quantity $F(t)$ is defined as

$$
\tilde{F}(\omega)=\int_{-T / 2}^{T / 2} \exp (-i \omega t) F(t) \mathrm{d} t
$$


Correspondingly, the inverse transformation is

$$
F(t)=\int_{-\infty}^{\infty} \exp (i \omega t) \tilde{F}(\omega) \frac{\mathrm{d} \omega}{2 \pi} .
$$

The finite-time $\delta$ function is given by

$$
2 \pi \delta_{T}(\omega)=\int_{-T / 2}^{T / 2} \exp (-i \omega t) \mathrm{d} t
$$

and therefore is finite: $2 \pi \delta_{T}(0)=T$.

The following is a brief derivation of Eq. (55). Substitution of the particular form of the correlation function [Eq. (53)] into the general formula [Eq. (39)] leads to

$$
\begin{aligned}
C_{V}\left(\omega, \omega^{\prime}\right)= & \sum_{m=-\infty}^{\infty} \sum_{n=-\infty}^{\infty} d_{m} d_{n}{ }^{*} \exp [-i(m-n) \gamma] \\
& \times \tilde{Z}\left(\omega-\Omega_{m}\right) \tilde{Z}\left(\omega^{\prime}-\Omega_{n}\right)^{*} \\
& \times 2 \pi q \sum_{p=-\infty}^{\infty} I_{p} \delta_{T}\left(\omega-\omega^{\prime}+\Omega_{m-n+p}\right) .
\end{aligned}
$$

For small frequency separation $\left(\left|\omega-\omega^{\prime}\right| \ll \Omega\right)$, the $\delta$ function on the right-hand side of Eq. (A4) can be approximated as

$$
\delta_{T}\left(\omega-\omega^{\prime}+\Omega_{m-n+p}\right) \approx \delta_{n-m, p} \delta_{T}\left(\omega-\omega^{\prime}\right) .
$$

This approximation is based on the observation ${ }^{11}$ that the finite-time $\delta$ function can be treated as a conventional Dirac $\delta$ function in the limit of large $T$. Therefore,

$$
\begin{aligned}
C_{V}\left(\omega, \omega^{\prime}\right)= & 2 \pi \delta_{T}\left(\omega-\omega^{\prime}\right) \\
& \times \sum_{m=-\infty}^{\infty} \sum_{n=-\infty}^{\infty} d_{m} d_{n}{ }^{*} \exp [-i(m-n) \gamma] \\
& \times q I_{n-m} \tilde{Z}\left(\omega-\Omega_{m}\right) \tilde{Z}\left(\omega-\Omega_{n}\right)^{*}, \quad(\mathrm{~A} 6)
\end{aligned}
$$

which leads to Eqs. (54) and (55).

\section{Appendix B. Correlation Function of Nonstationary Shot Noise}

Textbook derivations of the shot-noise correlation function are usually limited to stationary noise. ${ }^{16}$ Therefore here I present a derivation of the correlation function for nonstationary shot noise.

The shot noise can be described by a train of pulses with random arrival times $t_{k}$. (The pulses correspond to the electric current produced by individual electrons.) Let the total number of pulses within the observation time be $K$. Then the noisy photocurrent is given by

$$
J(t)=q \sum_{k=1}^{K} \delta\left(t-t_{k}\right)
$$

Not only are the arrival times random, but the total number of pulses is also random. Assume that there is an average $N$ for the total number of pulses, which is usually the case if the average photocurrent $I(t)$ is periodic and the observation time is much greater than the period.

The random nature of the photon arrival times is characterized by the probability density for pulses $n(t)$, which is proportional to the average photocurrent. ${ }^{17,18}$ In other words, the probability for a pulse to be detected within the interval $(t, t+\mathrm{d} t)$ is given by

$$
n(t) \mathrm{d} t=\frac{I(t)}{q N} \mathrm{~d} t .
$$

Therefore, averaging over the photon arrival times can be defined as averaging with the probability density $n(t)$. This averaging is denoted by an overbar as in the following example:

$$
\begin{aligned}
\overline{\delta\left(t-t_{k}\right)} & =\int_{-T / 2}^{T / 2} \delta\left(t-t_{k}\right) n\left(t_{k}\right) \mathrm{d} t_{k} \\
& =n(t) .
\end{aligned}
$$

The total number of pulses within any given time interval is not just random but also varies with time in a deterministic manner. As the time interval becomes larger, the deterministic time dependence becomes less important. For a large observation time $T$, the total number of pulses approach a Poisson distribution, even though the pulses are nonstationary. Then the probability of counting $K$ pulses within the observation time is

$$
p(K)=\frac{N^{K}}{K !} \exp (-N),
$$

where $N$ is the average number of pulses. This distribution is valid as long as the observation time is much greater than the modulation period. Therefore, averaging with respect to the total number of pulses can be defined as averaging with the probability $p(K)$. We denote this averaging by $\langle\ldots .$.$\rangle as in$ the following example:

$$
\langle K\rangle \equiv \sum_{K=0}^{\infty} K p(K)=N
$$

Thus, one can determine the expectation value for a random process that involves the nonstationary Poisson pulses by taking the average with respect to both $t_{k}$ and $K$. For example, the expectation value of the photocurrent can be found as

$$
\begin{aligned}
\mathbf{E}[J(t)] & =\left\langle q \sum_{k=1}^{K} \overline{\delta\left(t-t_{k}\right)}\right\rangle \\
& =\langle q K n(t)\rangle \\
& =q N n(t) .
\end{aligned}
$$

This result is equivalent to the definition of the probability density for the pulses [Eq. (B2)].

Consider now the correlation function of the non- 
stationary shot noise [Eq. (34)], which can be written as

$$
C_{I}\left(t, t^{\prime}\right)=\mathbf{E}\left[J(t) J\left(t^{\prime}\right)\right]-I(t) I\left(t^{\prime}\right) .
$$

The expectation value

$$
\mathbf{E}\left[J(t) J\left(t^{\prime}\right)\right]=\left\langle q^{2} \sum_{k=1}^{K} \sum_{m=1}^{K} \overline{\delta\left(t-t_{k}\right) \delta\left(t^{\prime}-t_{m}\right)}\right\rangle .
$$

It is convenient to separate the sum over diagonal $(k=m)$ and off-diagonal $(k \neq m)$ terms. First consider the sum over diagonal terms. A typical diagonal term gives rise to the following average:

$$
\begin{aligned}
\overline{\delta\left(t-t_{k}\right) \delta\left(t^{\prime}-t_{m}\right)} & =\int_{-T / 2}^{T / 2} \delta\left(t-t_{k}\right) \delta\left(t^{\prime}-t_{k}\right) n\left(t_{k}\right) \mathrm{d} t_{k} \\
& =n(t) \delta\left(t-t^{\prime}\right) .
\end{aligned}
$$

The number of such terms in the sum is equal to the total number of pulses $K$. Therefore, according to Eq. (B5), the average number of such terms is $N$.

Second, consider the sum over off-diagonal terms. In any such term, the photon arrival times are independent. Therefore,

$$
\begin{aligned}
\overline{\delta\left(t-t_{k}\right) \delta\left(t^{\prime}-t_{m}\right)} & =\overline{\delta\left(t-t_{k}\right)} \overline{\delta\left(t^{\prime}-t_{m}\right)} \\
& =n(t) n\left(t^{\prime}\right) .
\end{aligned}
$$

There are $\left(K^{2}-K\right)$ such terms in the sum. Thus the average number of these terms is

$$
\left\langle\left(K^{2}-K\right)\right\rangle \equiv \sum_{K=0}^{\infty}\left(K^{2}-K\right) p(K)=N^{2} .
$$

Combining Eqs. (B8)-(B11), we obtain the formula for the correlation function of the photocurrent:

$$
\begin{aligned}
\mathbf{E}\left[J(t) J\left(t^{\prime}\right)\right] & =q^{2} N n(t) \delta\left(t-t^{\prime}\right)+q^{2} N^{2} n(t) n\left(t^{\prime}\right) \\
& =q I(t) \delta\left(t-t^{\prime}\right)+I(t) I\left(t^{\prime}\right) .
\end{aligned}
$$

Then according to Eq. (B7) the correlation function of the noise is

$$
C_{I}\left(t, t^{\prime}\right)=q I(t) \delta\left(t-t^{\prime}\right)
$$

which in the frequency domain becomes

$$
\tilde{C}_{I}\left(\omega, \omega^{\prime}\right)=q \tilde{I}\left(\omega-\omega^{\prime}\right),
$$

where $\tilde{I}(\omega)$ is the Fourier transform of the average photocurrent.

I thank Fred Raab for suggesting the problem that led to this paper and Barry Barish for supporting this research project. I also thank Adrian Ottewill for checking some of the calculations, and Albrecht Rüdiger and Stan Whitcomb for discussions of the nonstationary shot noise. Finally, I thank Karen Gervais, Peter King, and Karen McCue for help dur- ing the preparation of this paper. This research was supported by the National Science Foundation under cooperative agreement PHY-9210038.

\section{References and Notes}

1. T. Niebauer, R. Schilling, K. Danzmann, A. Rüdiger, and W. Winkler, "Nonstationary shot noise and its effect on the sensitivity of interferometers," Phys. Rev. A 43, 5022-5029 (1991).

2. B. Meers and K. Strain, "Modulation, signal, and quantum noise in interferometers," Phys. Rev. A 44, 4693-4703 (1991).

3. N. Mio and K. Tsubono, "Observation of an effect due to nonstationary shot noise," Phys. Lett. A 164, 255-258 (1992).

4. M. B. Gray, A. J. Stevenson, H.-A. Bachor, and D. E. McClelland, "Harmonic demodulation of nonstationary shot noise," Opt. Lett. 18, 759-761 (1993).

5. P. Winzer, "Shot-noise formula for time-varying photon rates: a general derivation,” J. Opt. Soc. Am. B 14, 2424-2429 (1997).

6. T. T. Lyons, M. W. Regehr, and F. Raab, "Shot noise in gravitational-wave detectors with Fabry-Perot arms," Appl. Opt. 39, 6761-6770 (2000).

7. A. Abramovici, W. E. Althouse, R. W. Drever, Y. Gürsel, S. Kawamura, F. J. Raab, D. Shoemaker, L. Sievers, R. E. Spero, K. S. Thorne, R. E. Vogt, R. Weiss, S. E. Whitcomb, and M. E. Zucker, "LIGO: the Laser Interferometer GravitationalWave Observatory," Science 256, 325-333 (1992).

8. C. Bradaschia, R. Delfabbro, A. Divirgilio, A. Giazotto, H. Kautzky, V. Montelatici, D. Passuello, A. Brillet, O. Cregut, P. Hello, C. N. Mann, P. T. Manh, A. Marraud, D. Shoemaker, J. Y. Vinet, F. Barone, L. Difiore, L. Milano, G. Russo, J. M. Aguirregabiria, H. Bel, J. P. Duruisseau, G. Lederman, P. Tourrenc, M. Capozzi, M. Longo, M. Lops, I. Pinto, G. Rotoli, T. Damour, S. Bonazzola, J. A. Marck, Y. Gourghoulon, L. E. Holloway, F. Fuligni, V. Iafolla, and G. Natale, "The VIRGO project: a wide band antenna for gravitational-wave detection," Nucl. Instrum. Methods Phys. Res. A 289, 518-525 (1990).

9. K. Tsubono, "300-m laser interferometer gravitational wave detector (TAMA 300) in Japan" in Proceedings of the first Eduardo Amaldi Conference on Gravitational Wave Experiments, E. Coccia, G. Pizzella, and F. Ronga, eds. (World Scientific, Singapore, 1995), pp. 112-114.

10. R. Drever, J. Hall, F. Kowalski, J. Hough, G. Ford, A. Munley, and $\mathrm{H}$. Ward, "Laser phase and frequency stabilization using an optical resonator," Appl. Phys. B 31, 97-105 (1983).

11. A. Papoulis, Probability, Random Variables, and Stochastic Processes, 3rd ed. (WCB/McGraw-Hill, Boston, Mass., 1991).

12. M. Rakhmanov, "Dynamics of laser interferometric gravitational wave detectors," Ph.D. dissertation (California Institute of Technology, Pasadena, Calif., 2000).

13. J. S. Bendat and A. G. Piersol, Random Data. Analysis and Measurement Procedures, 2nd ed. (Wiley, Singapore, 1991).

14. This observation was made by F. Raab during a discussion of the nonstationary shot noise in the 40-m prototype of LIGO interferometers at Caltech, Pasadena, Calif., (1996).

15. K. S. Thorne, "Gravitational radiation," in Three Hundred Years of Gravitation, S. W. Hawking and W. Israel, eds. (Cambridge University, Cambridge, England, 1987), pp. 330-458.

16. W. Davenport and W. Root, Introduction to the Theory of Random Signals and Noise (McGraw-Hill, New York, 1958).

17. R. Loudon, Quantum Theory of Light, 2nd ed. (Oxford University, London, 1983).

18. L. Mandel and E. Wolf, Optical Coherence and Quantum Optics (Cambridge University, Cambridge, England, 1995). 